\title{
On Welch-Gong Transformation Sequence Generators
}

\author{
G. Gong and A.M. Youssef \\ Center for Applied Cryptographic Research, \\ Department of Combinatorics and Optimization, \\ University of Waterloo, \\ Waterloo, Ontario N2L 3G1, CANADA \\ \{ggong, a2youssef \}@cacr. math. uwaterloo.ca
}

\begin{abstract}
Welch-Gong (WG) transformation sequences are binary sequences of period $2^{n}-1$ with 2-level auto correlation. These sequences were discovered by Golomb, Gong and Gaal in 1998 and verified for $5 \leq n \leq 20$. Later on, No, Chung and Yun found another way to construct the WG sequences and verified their result for $5 \leq n \leq 23$. Dillon first proved this result for odd $n$ in 1998, and finally, Dobbertin and Dillon proved it for even $n$ in 1999. In this paper, we investigate a two-faced property of the WG transformation sequences for application in stream ciphers and pseudo-random number generators. One is to present randomness or unpredictability of the WG transformation sequences. The other is to exhibit the security property of the WG transformations regarded as Boolean functions. It is shown that the WG transformation sequences, in addition to the known 2-level auto correlation, have threelevel cross correlation with m-sequences, large linear ! span increasing exponentially with $n$ and efficient implementation. Thus this is the first type of pseudo-random sequences with good correlation and statistic properties, large linear span and efficient implementation. When the WG transformation are regarded as Boolean functions, it is proved that they have high nonlinearity. A criterion for whether the WG transformations regarded as Boolean functions are $r$-resilient is derived. It is shown that the WG transformations regarded as Boolean functions have large linear span (this concept will be defined in this paper) and high degree.
\end{abstract}

Key words: Stream cipher, pseudo-random sequence (number) generator, auto/cross correlation, linear span, Boolean function, non-linearity, $r$-resilient property.

\section{Introduction}

Pseudo-random sequences have been widely used in communications and cryptology. In order to guarantee that the pseudo-random sequence generators have good randomness or unpredictability, we have the following criteria:

- Long period

- Balance property (Golomb Postulate $1[5]$ )

D.R. Stinson and S. Tavares (Eds.): SAC 2000, LNCS 2012, pp. 217-232 2001.

(C) Springer-Verlag Berlin Heidelberg 2001 
- Run property (Golomb Postulate 2)

- $n$-tuple distribution

- Two-level auto correlation (Golomb Postulate 3)

- Low-level cross correlation

- Large linear span and smooth increased linear span profiles

In the last three years, the study of binary sequences with 2-level autocorrelation has made significant progress. The researchers [46[12]14|16] have found a number of new classes of binary sequences with 2-level auto correlation. In general, a pseudo-random sequence generator which generates sequences with 2-level auto correlation can be resistant to a correlation attack. However it is not easy to design a pseudo-random sequence generator which can generate sequences having both 2-level auto correlation and large linear span and be efficient to implement as well. Fortunately, it happened that one of classes of new sequences with 2-level auto correlation, so-called the Welch-Gong transformation sequences, possesses all these three properties. On the other hand, this type of sequences has period $2^{n}-1$. Any binary sequence of period $2^{n}-1$ is related to a function from the finite field $G F\left(2^{n}\right)$ to the finite field $G F(2)$. Thus it is automatically related to a Boolean function in $n$ variables. Thus there is a connection among binary sequences with period $2^{n}-1$, polynomial functions from $G F\left(2^{n}\right)$ to $G F(2)$ and Boolean functions in $n$ variables. Chang, Dai and Gong [1] tried to use this connection. I.e., they applied m-sequences with three-level cross correlation to construct Boolean functions with the maximal non-linearity. In [10], Gong and Golomb successfully tried again to utilize this connection. They applied tools in pseudo-random sequence design and analysis to analyze the S-boxes in DES (Data Encryption Standard). When they considered the relationship between sequences and functions, they realized that monomials, which correspond to $\mathrm{m}$ sequences, are not secure when used as component functions in block ciphers. This leads to a concept of linear span for polynomial functions introduced in their recent work [10. In this paper, we will investigate the Welch-Gong transformation sequences in a two-faced aspect. One is to present their randomness, i.e., auto correlation, cross correlation with $m$-sequences, the balance property, and linear span when we consider them as sequences. The other is to derive the nonlinearity, the resilient property, linear span and degree when they are regarded as Boolean functions.

This paper is organized as follows. In Section 2, we give the definition of Welch-Gong transformation sequences. In Section 3, we present the randomness properties of the Welch-Gong transformation sequences which include an irregular decimation property, statistic properties, cross correlation with m-sequences, the Hadamard transform, and the linear span. In Section 4, we derive the nonlinearity and a criterion for the resilient property (Note. Since any Welch-Gong transformation is balanced, so the correlation immunity property becomes the resilient property). In Section 5, we discuss linear span for the Welch-Gong transformations regarded as Boolean functions and show their degrees. Section 6 is a conclusion. All proofs omitted from this extended abstract can be found in the full paper [7]. 
We conclude this section by providing some preliminaries for sequence designs. The reader is referred to [5] for shift register sequences, [11] for the theory of finite field, and [19] and 18] for the motivation and the original definitions of nonlinearity and the resilient property for Boolean functions.

We will use the following notation throughout the paper:

$-\mathbb{F}_{q}=G F(q)$, a finite field with $q$ elements, and $\mathbb{F}_{q}^{*}$, the multiplication group of $\mathbb{F}_{q}$.

$-\mathbb{F}_{2}^{n}=\left\{\underline{\mathbf{x}}=\left(x_{0}, x_{1}, \cdots, x_{n-1}\right) \mid x_{i} \in \mathbb{F}_{2}\right\}$, a vector space over $\mathbb{F}_{2}$ of dimension $n$.

- $\underline{\mathbf{a}}=\left\{a_{i}\right\}$, a sequence over $\mathbb{F}_{2}$, i.e., $a_{i} \in \mathbb{F}_{2}$, is called a binary sequence. If $\underline{\mathbf{a}}$ is a periodic sequence with period $v$, then we also denote $\underline{\mathbf{a}}=\left(a_{0}, a_{1}, \cdots, a_{v-1}\right)$, an element in $\mathbb{F}_{2}^{v}$.

\section{A. Autocorrelation}

If $\underline{\mathbf{a}}=\left(a_{0}, a_{1}, \cdots, a_{p-1}\right)$ is a binary sequence with period $p$, its (periodic) autocorrelation function $C(\tau)$ is defined as

$$
C(\tau)=\sum_{i=0}^{p-1}(-1)^{a_{i}+a_{i+\tau}}, \tau=0,1, \cdots .
$$

Here $\tau$ is a phase shift of the sequence $\left\{a_{i}\right\}$.

Ideal (2-Level) Autocorrelation: If

$$
C(\tau)=\left\{\begin{array}{l}
p \quad \text { if } \tau \equiv 0 \bmod p \\
-1 \text { otherwise }
\end{array}\right.
$$

then we say that the sequence $\left\{a_{i}\right\}$ has the idea two-level autocorrelation function.

\section{B. Cross Correlation}

If $\underline{\mathbf{a}}=\left(a_{0}, a_{1}, \cdots, a_{p-1}\right)$ and $\underline{\mathbf{b}}=\left(b_{0}, b_{1}, \cdots, b_{p-1}\right)$ are two binary sequences with period $p$, their (periodic) cross correlation function $C_{\underline{\mathbf{a}}, \underline{\mathbf{b}}}(\tau)$ is defined as

$$
C_{\underline{\mathbf{a}}, \underline{\mathbf{b}}}(\tau)=\sum_{i=0}^{p-1}(-1)^{a_{i+\tau}+b_{i}}, \tau=0,1, \cdots .
$$

Here $\tau$ is a phase shift of the sequence $\left\{b_{i}\right\}$.

\section{Hamming Weight}

Let $H(s)=\left|\left\{0 \leq i<2^{n}-1 \mid s_{i}=1\right\}\right|$ if $s=\left\{s_{i}\right\}$ is a binary sequence with period $2^{n}-1$ and $H(s)=\left|\left\{x \in \mathbb{F}_{2^{n}} \mid s(x)=1\right\}\right|$ if $s=s(x)$ is a polynomial function from $\mathbb{F}_{2^{n}}$ to $\mathbb{F}_{2}$. In both cases, we call $H(s)$ the Hamming weight of $s$. For a positive integer $r=r_{0}+r_{1} 2+\cdots r_{n-1} 2^{n-1}, r_{i} \in \mathbb{F}_{2}, H(r)=\left|\left\{0 \leq i<n \mid r_{i}=1\right\}\right|$ is also called the Hamming weight of the integer $r$. 


\section{Definition of Welch-Gong Transformation Sequence Generators}

In this section, we will give the definition of the Welch-Gong transformation sequence generators. Hence after, we set $n \neq 0$ mod 3 .

Let $g(x)=x+x^{q_{1}}+x^{q_{2}}+x^{q_{3}}+x^{q_{4}}, x \in \mathbb{F}_{2^{n}}$, where $q_{i}$ are defined by

$$
\begin{aligned}
& q_{1}=2^{k}+1 \\
& q_{2}=2^{2 k-1}+2^{k-1}+1 \\
& q_{3}=2^{2 k-1}-2^{k-1}+1 \text { and } \\
& q_{4}=2^{2 k-1}+2^{k}-1
\end{aligned}
$$

where $n=3 k-1$ and

$$
\begin{aligned}
& q_{1}=2^{k-1}+1 \\
& q_{2}=2^{2 k-2}+2^{k-1}+1 \\
& q_{3}=2^{2 k-2}-2^{k-1}+1 \text { and } \\
& q_{4}=2^{2 k-1}-2^{k-1}+1
\end{aligned}
$$

where $n=3 k-2$. Then a function, say $f(x)$, from $\mathbb{F}_{2^{n}}$ to $\mathbb{F}_{2}$ defined by

$$
f(x)=\operatorname{Tr}(g(x+1)+1), x \in \mathbb{F}_{2^{n}}
$$

is called the Welch-Gong transformation of $\operatorname{Tr}(g(x))$, or the WG transformation for short.

Let $\alpha$ be a primitive element of $\mathbb{F}_{2^{n}}$. Let $\underline{\mathbf{a}}=\left\{a_{i}\right\}$ and $\underline{\mathbf{b}}=\left\{b_{i}\right\}$ whose elements are given by

$$
a_{i}=\operatorname{Tr}\left(g\left(\alpha^{i}\right)\right), b_{i}=f\left(\alpha^{i}\right)=\operatorname{Tr}\left(g\left(\alpha^{i}+1\right)+1\right), i=0,1, \cdots .
$$

Then $\underline{\mathbf{b}}$ is called a Welch-Gong transformation sequence of $\underline{\mathbf{a}}$, or WG sequence for short.

Any function from $\mathbb{F}_{2^{n}}$ to $\mathbb{F}_{2}$ is related to a Boolean function (we will discuss an exact conversion of these two representations in Section 4). From a WG transformation, we have two types of pseudo-random sequence generators. One is WG sequences themselves. The other is to apply WG transformations regarded as Boolean functions to operate on a set of LFSRs for generating sequences. I.e., applying the WG transformations regarded as Boolean functions as combining functions or filtering functions in combinatorial function generators or filtering generators 17. We refer to these two modes as WG sequence generators.

Remark 1. In fact, the transform given in (3) can be applied to any function from $\mathbb{F}_{2^{n}}$ to $\mathbb{F}_{2}$. But till now, we haven't found another type of $g(x)$ such that its WG transformation sequence has 2-level auto correlation. So, we restrict ourselves to this specific $g(x)$. 


\section{Randomness of WG Sequences}

In this section, we will discuss the randomness properties of WG sequences, including their decimation, auto/cross correlation, statistic properties, and linear span.

\section{A. Decimation Property}

Lemma 1. Let $\alpha$ be a primitive element of $\mathbb{F}_{2^{n}}$ and $\underline{\mathbf{b}}$ be the $W G$ sequence of $\underline{\mathbf{a}}$. Then elements of $\underline{\mathbf{b}}$ can be obtained by operating an irregular decimation on $\underline{\mathbf{a}}$ as follows: $b_{0}=a_{0}$ for $i>0$,

$$
b_{i}= \begin{cases}a_{\tau(i)} & \text { if } n \text { even } \\ a_{\tau(i)}+1 & \text { if } n \text { odd }\end{cases}
$$

where $\tau(i)$ is determined by

$$
\alpha^{\tau(i)}=\alpha^{i}+1
$$

Proof. From the definition, $a_{0}=\operatorname{Tr}(g(1))=n$ and $b_{0}=\operatorname{Tr}(g(0)+1)=n$. Thus $a_{0}=b_{0}$. For $i>0$, if $n$ is even, we have

$$
\begin{aligned}
b_{i} & =\operatorname{Tr}\left(g\left(\alpha^{i}+1\right)+1\right)=\operatorname{Tr}\left(g\left(\alpha^{i}+1\right)\right)+n \\
& =\operatorname{Tr}\left(g\left(\alpha^{i}+1\right)\right)=a_{\tau(i)}, i=0,1, \cdots .
\end{aligned}
$$

Similarly, if $n$ is odd, we have

$$
\begin{aligned}
b_{i} & =\operatorname{Tr}\left(g\left(\alpha^{i}+1\right)+1\right)=\operatorname{Tr}\left(g\left(\alpha^{i}+1\right)\right)+n \\
& =\operatorname{Tr}\left(g\left(\alpha^{i}+1\right)\right)=a_{\tau(i)}+1, i=0,1, \cdots .
\end{aligned}
$$

Thus the assertion is established.

Remark 2. This lemma shows that the WG sequence $\underline{\mathbf{b}}$ can be obtained by an irregular decimation from $\underline{\mathbf{a}}$ where the decimation is determined by (5). Note that $\underline{\mathbf{a}}$ is a 5 -term sequence and it can be generated by using five linear feedback shift registers and one AND gate. This property of the WG sequences allows them to have an efficient implementation for small $n$ by operating decimation on $\underline{\mathbf{a}}$ together with a table look-up.

\section{B. Auto Correlation, 2-Tuple Distribution, and Balance Property}

Proposition 1. Let $\underline{\mathbf{b}}$ be a WG sequence defined by (4). Then $\underline{\mathbf{b}}$ is a binary sequence of period $2^{n}-1$ with (ideal) 2-level auto correlation.

The proof of this proposition can be found in [4].

Remark 3. This result was first discovered by Golomb, Gong and Gaal in [12] and verified for $5 \leq n \leq 20$. Later on, No et al [16], found another way to construct the WG sequences and verified their result for $5 \leq n \leq 23$. Dillon proved it for the odd $n$ case [2], and finally, Dobbertin and Dillon proved it for the even $n$ case [4] which completely established Proposition 1]. 
Note. $\mathbf{a}$ is also a 2-level auto correlation sequence. See 124 .

Let $\underline{\mathbf{s}}$ be a binary sequence of period $2^{n}-1$. Assume that $1 \leq t \leq n$. In every period of $\underline{\mathbf{s}}$, if each nonzero $t$-tuple $\left(c_{1}, c_{2}, \cdots, c_{t}\right) \in F_{2}^{t}$ occurs $2^{n-t}$ times and zero $t$-tuple $(0, \cdots, 0)$ occurs $2^{n-t}-1$ times, then we say that the sequence has an ideal t-tuple distribution.

Proposition 2. Any WG sequence has ideal 2-tuple distribution and it is balanced (i.e., in every period $2^{n}-1$, zeros occur $2^{n-1}-1$ times and ones occur $2^{n-1}$ times).

Proof. Let $\underline{\mathbf{b}}$ be a WG sequence of period $2^{n}-1$. Since $\underline{\mathbf{b}}$ is a 2 -level auto correlation sequence, then for any shift of $\underline{\mathbf{b}}$, say $L^{\tau}(\underline{\mathbf{b}})=\left(b_{\tau}, b_{\tau+1}, \cdots\right)$, we have

$$
\left|\left\{0 \leq k<2^{n}-1 \mid\left(b_{k}, b_{k+\tau}\right)=(i, j)\right\}\right|= \begin{cases}2^{n-2}-1 & \text { if } i=j=0 \\ 2^{n-2} & \text { otherwise }\end{cases}
$$

where $i, j \in \mathbb{F}_{2}$. When $\tau=1$ it establishes the first assertion. The second result follows from the first one. (For a detail proof, please see the full paper.)

\section{Hadamard Transform and Cross Correlation}

Let $f(x)$ be a function from $\mathbb{F}_{2^{n}}$ to $\mathbb{F}_{2}$. Then the Hadamard transform of $f(x)$ is defined by

$$
\hat{f}(\lambda)=\sum_{x \in \mathbb{F}_{2^{n}}}(-1)^{\operatorname{Tr}(\lambda x)+f(x)} .
$$

Property 1. Let $S_{v}(x)=\operatorname{Tr}\left(x^{v}\right)$. Let $n=2 m+1$ be odd and $v=2^{t}+1$ with $\operatorname{gcd}(t, n)=1$. Then the Hadamard transform of $S_{2^{t}+1}(x)$ is given by

$$
\hat{S}_{2^{t}+1}(\lambda)= \begin{cases}0 & \text { if } \operatorname{Tr}(\lambda)=0 \\ \pm 2^{m+1} & \text { if } \operatorname{Tr}(\lambda)=1\end{cases}
$$

This result is established by Gold [8] in 1968 .

Theorem 1. For odd $n$, let $g(x)$ be defined as in Section 2.1, and let $f(x)$ be the Welch-Gong transform of $\operatorname{Tr}(g(x))$, i.e., $f(x)=\operatorname{Tr}(g(x+1)+1)$. Then $\hat{f}(\lambda)$, the Hadamard transform of $f(x)$, is given by

$$
\hat{f}(\lambda)=\hat{S}_{2^{t}+1}\left(\lambda^{c}\right),
$$

where

$$
c=d^{-1} \text { and } d=2^{2 t}-2^{t}+1 \text { for } 3 t \equiv 1 \bmod n .
$$

Proof. Here we only give a link with the source for our proof. The linear span of WG sequences are give in [12] by Golomb, Gong and Gaal. Later on, No, Chung and Yin found another way to generate the WG sequences in [16] which 
is verified by experimental results. A few months later, Dobbertin proved that the sequences generated in [16] are WG sequences in [3] through these two types of sequences having the same linear span. Dillon proved that the sequences generated by No-Chung-Yin have 2-level autocorrelation for $n$ odd by establishing the Hadamard transform of an No-Chung-Yin sequence is equal to the value of the right hand of equation (10). Thus the result follows from the above link.

From Theorem 1 and Property 1, we now have the following corollaries.

Corollary 1 (Function Version). Let $n=2 m+1$ odd, and let $f(x)$ be the Welch-Gong transformation function. Then the Hadamard transform of $f(x)$ is given by

$$
\hat{f}(\lambda)= \begin{cases}0 & \text { if } \operatorname{Tr}\left(\lambda^{c}\right)=0, \\ \pm 2^{m+1} & \text { if } \operatorname{Tr}\left(\lambda^{c}\right)=1,\end{cases}
$$

where $c$ is defined in (11) in Theorem 1.

Proof. Applying Property 1 to Theorem 1, the result follows.

Corollary 2 (Sequence Version). Let $\alpha$ be a primitive element of $\mathbb{F}_{2^{n}}$ and $f(x)$ be a Welch-Gong transformation function. Let $\underline{\mathbf{a}}=\left\{a_{i}\right\}$ be an m-sequence whose elements are defined by

$$
a_{i}=\operatorname{Tr}\left(\alpha^{i}\right), i=0,1, \cdots
$$

and $\underline{\mathbf{b}}=\left\{b_{i}\right\}$ be the $W G$ sequence whose elements are given by (4)). Then $C_{\underline{\mathbf{a}}, \underline{\mathbf{b}}}(\tau)$, the cross correlation function between $\underline{\mathbf{a}}$ and $\underline{\mathbf{b}}$, is determined by

$$
C_{\underline{\mathbf{a}}, \underline{\mathbf{b}}}(\tau)= \begin{cases}-1 & \text { if } \operatorname{Tr}\left(\alpha^{\tau c}\right)=0, \\ -1 \pm 2^{m+1} & \text { if } \operatorname{Tr}\left(\alpha^{\tau c}\right)=1,\end{cases}
$$

where $c$ is defined in (11) in Theorem 1. I.e., the cross correlation function between $\underline{\mathbf{a}}$ and $\underline{\mathbf{b}}$ are three-valued.

D. Linear Span of WG Sequences

The linear span of a sequence is defined to be the shortest length of the linear feedback shift registers which generate the sequence. Sequence with large linear span are resistant to attacks arising from employing the Berlekamp-Massey algorithm [15].

Proposition 3. Let $\underline{\mathbf{b}}$ be a $W G$ sequence of period $2^{n}-1$ and $L S(\underline{\mathbf{b}})$ represent its linear span. Then

$$
L S(\underline{\mathbf{b}})=n\left(2^{\lceil n / 3\rceil}-3\right) .
$$

A proof for this result is given in Section 5. From Proposition 3 it is clear that the linear span of the WG sequences of period $2^{n}-1$ increases exponentially with $n$.

Remark 4. WG sequences of period $2^{n}-1$ are the first type of binary sequences of period $2^{n}-1$ which have the balance property, ideal 2-tuple distribution, 2level auto correlation, three-level cross correlation with m-sequences, linear span increased exponentially in $n$. 


\section{Non-linearity and Resilient Property of Welch-Gong Transformations}

In this section, we will derive the non-linearity and the resilient property for the WG transformations when they are regarded as Boolean functions. First, we need to develop a result on the conversion of a polynomial function (from $\mathbb{F}_{2^{n}}$ to $\mathbb{F}_{2}$ ) to a Boolean function in $n$ variables.

\subsection{Isomorphism between $\mathbb{F}_{2^{n}}$ and $\mathbb{F}_{2}^{n}$}

Since the finite field $\mathbb{F}_{2^{n}}$ can be regarded as a vector space of $n$ dimension, then we have a linear space structure for $\mathbb{F}_{2^{n}}$. Let $B=\left\{\alpha_{0}, \alpha_{1}, \cdots, \alpha_{n-1}\right\}$ be a basis of $\mathbb{F}_{2^{n}}$ over $\mathbb{F}_{2}$, then $\forall x \in F_{2^{n}}$, we have

$$
x=x_{0} \alpha_{0}+x_{1} \alpha_{1}+\cdots+x_{n-1} \alpha_{n-1}, x_{i} \in F_{2} .
$$

Let

$$
\delta: x \mapsto \underline{\mathbf{x}}=\left(x_{0}, x_{1}, \cdots, x_{n-1}\right)
$$

then $\delta(x)$ is an isomorphism between $\mathbb{F}_{2^{n}}$ and $\mathbb{F}_{2}^{n}$ when both of them are regarded as vector spaces.

Let $f(x)$ be a function from $\mathbb{F}_{2^{n}}$ to $\mathbb{F}_{2}$. Then $f(x)$ defines a Boolean function in the following way:

$$
f(x)=f\left(x_{0} \alpha_{0}+\cdots+x_{n-1} \alpha_{n-1}\right)=f_{B}\left(x_{0}, x_{1}, \cdots, x_{n-1}\right)
$$

Note that $f$ and $f_{B}$ in the above identity might not be same. We will write $f_{B}\left(x_{0}, x_{1}, \cdots, x_{n-1}\right)=f\left(x_{0}, x_{1}, \cdots, x_{n-1}\right)$ for short if it will not cause any confusion in the context.

Remark 5. For a given Boolean function in $n$ variables, we can obtain its polynomial representation which is a function from $\mathbb{F}_{2^{n}}$ to $\mathbb{F}_{2}$ by using so-called the Fourier transform, see [10].

\subsection{Non-linearity of WG Transformations}

Let $f(\underline{\mathbf{x}}), \underline{\mathbf{x}}=\left(x_{0}, x_{1}, \cdots, x_{n-1}\right) \in F_{2^{n}}$, be a Boolean function. A Boolean function $a(\underline{\mathbf{x}})$ is said to be affine if

$$
a(\underline{\mathbf{x}})=\sum_{i=0}^{n-1} w_{i} x_{i}+c, w_{i} \in \mathbb{F}_{2}, c \in \mathbb{F}_{2} .
$$

Let

$$
A=\left\{\sum_{i} w_{i} x_{i}+c \mid w_{i} \in \mathbb{F}_{2}, c \in \mathbb{F}_{2}\right\}
$$


I.e., $A$ is the set consisting of all affine Boolean functions in $n$ variables. Then the non-linearity of $f(\underline{\mathbf{x}})$, denoted by $N_{f}$, is defined as

$$
N_{f}=\min _{a \in A} d(f, a)
$$

where $d(f, a)=\left|\left\{\underline{\mathbf{x}} \in F_{2}^{n} \mid f(\underline{\mathbf{x}}) \neq a(\underline{\mathbf{x}})\right\}\right|$ which is called a distance of $f$ from $a$.

Remark 6. From the definition of the distance, we have $d(f, a)=H(f+a)$, where $H(f+a)$ is the Hamming weight of $f+a$ defined in Section 1 .

Note that

$$
d(f, a)=\left|\left\{x \in \mathbb{F}_{2^{n}} \mid f(x) \neq a(x)\right\}\right|
$$

where $f(x)$ and $a(x)$ are polynomial forms of the Boolean functions $f(\underline{\mathbf{x}})$ and $a(\underline{\mathbf{x}})$ respectively. I.e., the distance of $f$ from $a$ is not changed whenever either a Boolean form or a polynomial form are applied.

Let

$$
\hat{f}(\underline{\mathbf{w}})=\sum_{\underline{\mathbf{x}} \in \mathbb{F}_{2}^{n}}(-1)^{\mathbf{w} \cdot \underline{\mathbf{x}}+f(\underline{\mathbf{x}})}
$$

which is called the Walsh transform of the Boolean function $f$ in the literature.

Theorem 2. Let $n=2 m+1$ odd. Let $f(x)$ be the Welch-Gong transformation defined by (3). Let $f(\underline{\mathbf{x}})$ be the Boolean form of $f(x)$. Then the non-linearity of $f(\underline{\mathbf{x}})$, denoted by $N_{f}$, is given by

$$
N_{f}=2^{n-1}-2^{m} .
$$

In order to prove Theorem 2, we need the following two lemmas whose proofs can be found in the full paper.

Lemma 2. Let $f(\underline{\mathbf{x}})$ be a Boolean function and $a(\underline{\mathbf{x}})$ be a linear Boolean function. Let $f(x)$ and $a(x)$ be a polynomial representation of $f(\underline{\mathbf{x}})$ and $a(\underline{\mathbf{x}})$ respectively.

1. $a(\underline{\mathbf{x}})=\sum_{i} w_{i} x_{i}=\underline{\mathbf{w}} \cdot \underline{\mathbf{x}}$ where $\underline{\mathbf{w}}=\left(w_{0}, w_{1}, \cdots, w_{n-1}\right) \in \mathbb{F}_{2}^{n}$. Moreover, there exists some $\lambda \in \mathbb{F}_{2^{n}}$ such that $a(\underline{\mathbf{x}})=\operatorname{Tr}(\lambda x)$.

2. The Hadamard transform of $f(x)$ and the Walsh transform of $f(\underline{\mathbf{x}})$ have the following relation:

$$
\hat{f}(\underline{\mathbf{w}})=\hat{f}(\lambda), \underline{\mathbf{w}} \in \mathbb{F}_{2}^{n}, \lambda \in \mathbb{F}_{2^{n}}
$$

where $\underline{\mathbf{w}} \cdot \underline{\mathbf{x}}=\operatorname{Tr}(\lambda x)$.

3. The Hadamard transform of $f$ and the distance of $f(\underline{\mathbf{x}})$ from a $(\underline{\mathbf{x}})$ are related by

$$
\hat{f}(\lambda)=2^{n}-2 d(f, a)
$$

where $\lambda$ is the same as above. Or equivalently,

$$
d(f, a)=\frac{2^{n}-\hat{f}(\lambda)}{2} .
$$


Lemma 3. Let $f(\underline{\mathbf{x}})$ be a function from $\mathbb{F}_{2}^{n}$ to $\mathbb{F}_{2}$. Let $a(\underline{\mathbf{x}})$ be a linear function from $\mathbb{F}_{2}^{n}$ to $\mathbb{F}_{2}$. Then $d(f, a+1)=2^{n}-d(f, a)$. Moreover $d(f, a+1)=\frac{2^{n}+\hat{f}(\lambda)}{2}$ where $\lambda$ satisfies that $a(\underline{\mathbf{x}})=\operatorname{Tr}(\lambda x)$.

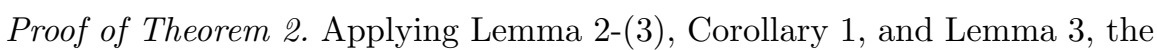
assertion follows. (A detailed proof is included in the full paper.)

Remark 7. Chang, Dai and Gong [1] discussed how to construct Boolean functions with the maximal non-linearity in terms of binary m-sequences with threevalued cross correlation. In particular, they discussed the case $\operatorname{Tr}\left(x^{r}\right)$ for some special choices of $r$. Gong and Golomb [10], pointed out that the monomial functions $\operatorname{Tr}\left(x^{r}\right)$ are not secure when used as combining functions or filtering functions in stream cipher systems or block cipher modes, because they correspond to m-sequences. However, the WG transformations are not monomials. We will come back to this question in the next section.

\subsection{The Resilient Property of WG Transformations}

Let $f(\underline{\mathbf{x}}), \underline{\mathbf{x}} \in F_{2}^{n}$, be a Boolean function. For $r>0, f(\underline{\mathbf{x}})$ is said to be $r$-order correlation immune if

$$
\hat{f}(\underline{\mathbf{w}})=0 \text { for all } \underline{\mathbf{w}} \in \mathbb{F}_{2}^{n}: 1 \leq H(\underline{\mathbf{w}}) \leq r .
$$

This definition comes from the result obtained by Xiao and Massey [20] which is equivalent to Siegenthaler's original definition [18. If $f(\underline{\mathbf{x}})$ satisfies (15) and $f(\underline{\mathbf{x}})$ is balanced, i.e., $H(f(\underline{\mathbf{x}}))=2^{n-1}$. Then $f(\underline{\mathbf{x}})$ is said to be $r$-resilient.

Let

$$
D=\left\{x \in \mathbb{F}_{2^{*}}^{*} \mid \operatorname{Tr}\left(x^{c}\right)=0\right\}
$$

where $c$ is defined in Theorem 1 . Then $|D|=2^{n-1}-1$. Recall that $\left\{\alpha_{0}, \alpha_{1}\right.$, $\left.\cdots, \alpha_{n-1}\right\}$ is the basis of $\mathbb{F}_{2^{n}}$ over $\mathbb{F}_{2}$. Let

$$
R=\left\{\left(\operatorname{Tr}\left(\lambda \alpha_{0}\right), \cdots, \operatorname{Tr}\left(\lambda \alpha_{n-1}\right)\right) \in \mathbb{F}_{2}^{n} \mid \lambda \in D\right\} .
$$

Theorem 3. With the above notation. Let $n$ be odd. Let $f(\underline{\mathbf{x}})$ be the Boolean form of the WG transformation $f(x)=\operatorname{Tr}(g(x+1)+1)$ defined by (3). Then $f(\underline{\mathbf{x}})$ is $r$-resilient if and only if all vectors in $W_{r}=\{\underline{\mathbf{w}} \mid 1 \leq H(\underline{\mathbf{w}}) \leq r\}$ appear in R. I.e., $W_{r} \subset R$.

Proof. Applying Lemma 2 we have $\hat{f}(\underline{\mathbf{w}})=\hat{f}(\lambda)$ where

$$
a(\underline{\mathbf{x}})=\sum_{i=0}^{n-1} w_{i} x_{i}=\operatorname{Tr}(\lambda x) .
$$

Notice that

$$
\lambda x=\lambda \sum_{i} x_{i} \alpha_{i} \Longrightarrow \operatorname{Tr}(\lambda x)=\sum_{i=0}^{n-1} \operatorname{Tr}\left(\lambda \alpha_{i}\right) x_{i} .
$$


Combining with (18), we have $w_{i}=\operatorname{Tr}\left(\lambda \alpha_{i}\right), 0 \leq i<n$. According to Corollary $1, \hat{f}(\lambda)=0$ if and only if $\operatorname{Tr}\left(\lambda^{c}\right)=0$ where $c$ is defined by 11). Together with the definition of the resilient property, the assertion follows.

Note. Theorem 3 provides a method to find $r$-resilient WG transformations regarded as Boolean functions.

In the following we will derive that any WG-transformation regarded as a Boolean function with 1-resilient property is always possible by a proper basis conversion.

Theorem 4. Let $f(x)$ be a WG-transformation. Then there exists at least one basis of $\mathbb{F}_{2^{n}}$ such that the Boolean function representation of $f(x)$ under this basis is 1-resilient

Proof. First we state the following claim whose proof can be found in the full paper.

Claim. There are $n$ linearly independent vectors in $R$, defined by (17).

Therefore we can assume that $\left\{\alpha_{0}, \cdots, \alpha_{n-1}\right\}$ is a subset of $R_{\underline{\alpha}}$ which is linearly independent over $\mathbb{F}_{2}$. Let $A$ be an $n \times n$ matrix with row vectors $\alpha_{i}, i=$ $0, \ldots, n-1$. Let $B=A^{-1}$ and let $\beta_{j}$ denote the $j$ th column vector of $B, j=$ $0, \cdots, n-1$. Then $\underline{\beta}=\left\{\beta_{0}, \cdots, \beta_{n-1}\right\}$ is a basis of $\mathbb{F}_{2^{n}}$. Then $R_{\underline{\beta}}$ is given by

$$
R_{\underline{\beta}}=\left\{\sigma_{\underline{\alpha}}(\lambda) B \mid \lambda \in D\right\} .
$$

Therefore the row vectors of $B A=A^{-1} A=I_{n}$, the identity matrix, belong to $R_{\beta}$. Hence $f_{\beta}\left(x_{0}, \cdots, x_{n-1}\right)$, the Boolean representation of $f(x)$ under the basis $\underline{\beta}$, is a 1 -resilient function.

\section{Linear Span and Degree of WG Transformations}

Let $f(x)=\sum_{i} c_{i} x^{i}$ be a polynomial function from $\mathbb{F}_{2^{n}}$ to $\mathbb{F}_{2}$. Let $I=\{0 \leq i \leq$ $\left.2^{n}-1 \mid c_{i} \neq 0\right\}$. The algebraic degree of $f(x)$, denoted as $\operatorname{alg}(f)$, is defined as

$$
\operatorname{alg}(f)=\max _{\{i \in I\}} \operatorname{alg}\left(x_{i}\right) \text { where } \operatorname{alg}\left(x_{i}\right)=H(i) .
$$

Let $f(\underline{\mathbf{x}})$ be the Boolean form of $f(x)$ and denote the degree of $f(\underline{\mathbf{x}})$ as $\operatorname{deg}(f(\underline{\mathbf{x}}))$.

Fact 1 The algebraic degree of $f(x)$, a polynomial function from $\mathbb{F}_{2^{n}}$ to $\mathbb{F}_{2}$, is equal to the degree of the Boolean form of the function. I.e., alg $(f)=\operatorname{deg}((f(\underline{\mathbf{x}}))$.

Linear span of $f(x)$ is said to be the number of non-zero coefficients in $f(x)=$ $\sum_{i} c_{i} x^{i}$, which is introduced by Gong and Golomb in 10 . We denote it as $L S(f(x))$ or simply $L S(f)$ if the context is clear. I.e., $L S(f)=|I|$.

Note. The linear span of a polynomial function from $\mathbb{F}_{2^{n}}$ to $\mathbb{F}_{2}$ is equal to the linear span of the sequence corresponding to the function. 
Let $f(\underline{\mathbf{x}})$ be a Boolean function, we will define the linear span of the Boolean function $f(\underline{\mathbf{x}})$ in terms of its polynomial representations. Let

$$
\Pi=\left\{\text { all bases of } \mathbb{F}_{2^{n}} \text { over } \mathbb{F}_{2}\right\} .
$$

For $B=\left\{\alpha_{0}, \cdots, \alpha_{n-1}\right\} \in \Pi$, we denote $f_{B}(x)$ a polynomial representation of $f(\underline{\mathbf{x}})$ with respect to the basis $B$. We define a linear span of $f(\underline{\mathbf{x}})$, denoted by $L S(f(\underline{\mathbf{x}}))$, as

$$
L S(f(\underline{\mathbf{x}}))=\min _{B \in \Pi} L S\left(f_{B}(x)\right) .
$$

Note that for a given polynomial function $f(x)$, the linear span of any Boolean representation of $f(x)$ is equal to the linear span of $f(x)$ itself. We will write this observation as a lemma for later reference.

Lemma 4. With the above notation. Let $f(x)$ be a polynomial function of $\mathbb{F}_{2^{n}}$ to $\mathbb{F}_{2}$. Let $f_{B}(\underline{\mathbf{x}})$ be its Boolean form with respect to a basis $B$ of $\mathbb{F}_{2^{n}}$ over $\mathbb{F}_{2}$. Then $L S\left(f_{B}(\underline{\mathbf{x}})\right)=L S(f(x))$ for all $B \in \Pi$.

As Youssef and Gong pointed it out in their recent work 21], the polynomial representation a complicated Boolean function might be just a monomial function (here monomial means that it has only one trace term in (19) which is different from the concept of the ordinary monomial which only has exactly one term). Thus a Boolean function must have a large linear span so that it can be resistant to the interpolation attack. In the following, we will show that the linear span of the Boolean forms of the WG transformations increases exponentially with $n$.

We have the following result whose proof can be found in [12].

Proposition 4. Let $f(x)=\operatorname{Tr}(g(x+1)+1)$ be the $W G$ transformation defined by (3), then

$$
f(x)=\sum_{i \in I} \operatorname{Tr}\left(x^{i}\right)
$$

where $I=I_{1} \cup I_{2}$ for $n=3 k-1$, where

$$
\begin{aligned}
& I_{1}=\left\{2^{2 k-1}+2^{k-1}+2+i \mid 0 \leq i \leq 2^{k-1}-3\right\}, \text { and } \\
& I_{2}=\left\{2^{2 k}+3+2 i \mid 0 \leq i \leq 2^{k-1}-2\right\}
\end{aligned}
$$

and where $I=\{1\} \cup I_{3} \cup I_{4}$ for $n=3 k-2$, where

$$
\begin{aligned}
& I_{3}=\left\{2^{k-1}+2+i \mid 0 \leq i \leq 2^{k-1}-3\right\}, \text { and } \\
& I_{4}=\left\{2^{2 k-1}+2^{k-1}+2+i \mid 0 \leq i \leq 2^{k-1}-3\right\} .
\end{aligned}
$$

Moreover, in each case, all the elements in I belong to distinct cyclotomic cosets modulo $2^{n}-1$.

Note that the trace functions appeared in (19) depend on the coset size of $i$ in $I$. 
Theorem 5. Let $f(x)=\operatorname{Tr}(g(x+1)+1)$ be the WG transformation defined by (3), then $L S(f(x))$, the linear span of $f$, is given by $L S(f(x))=n\left(2^{\lceil n / 3\rceil}-3\right)$.

Proof. According to Fact 4, all numbers in $I$ belong to different cyclotomic cosets modulo $2^{n}-1$ and $|I|=2^{k}-3$. So we only need to show that any coset containing a number in $I$ has full size $n$. Here we only give a proof for $n=3 k-1$ and $s \in I_{1}$, since proofs for the other cases are similar. Note that for $s \in I_{1}$, the binary representation of $s$ has the following pattern

Index $\quad 01 \cdots k-1 k \cdots 2 k-22 k-12 k \cdots 3 k-2=n-1$

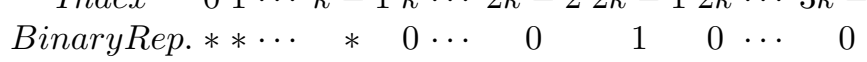

where $^{*}$ can take any value from $\{0,1\}$. Let $C_{s}$ be a coset containing $s$, then

$$
C_{s}=\left\{s, s 2, \cdots, s 2^{n_{s}-1}\right\}
$$

where $n_{s}$ is the smallest integer satisfying $s 2^{n_{s}} \equiv s \bmod 2^{n}-1$. According to (22), $n_{s}=n$. I.e., $C_{s}$ has full size $n$. Thus for each $s \in I$, we have the trace function appeared in Fact 4 is the trace function from $\mathbb{F}_{2^{n}}$ to $\mathbb{F}_{2}$. Thus $\operatorname{LS}\left(\operatorname{Tr}\left(x^{i}\right)\right)=n$ for each $i \in I$. Therefore the result follows.

Proof of Proposition 3 in Section 3. Since the linear span of a WG sequence $\underline{\mathbf{b}}$ is equal to the linear span of the corresponding WG transformation $f$. I.e., we have $L S(\underline{\mathbf{b}})=L S(f)$. Applying Theorem [5, the result follows.

Theorem 6. Let $f(x)$ be the WG transformation defined by (3). Then the linear span of any Boolean form of $f(x)$ is equal to the linear span of $f(x)$. I.e., $L S(f(\underline{\mathbf{x}}))=n\left(2^{\lceil n / 3\rceil}-3\right)$.

Proof. The result follows from Lemma 4 and Theorem 5.

Theorem 7. Let $f(x)=\operatorname{Tr}(g(x+1)+1)$ be the $W G$ transformation defined by (3) and $f(\underline{\mathbf{x}})$ be its Boolean form. Then $\operatorname{deg}(f(\underline{\mathbf{x}}))$, the degree of $f(\underline{\mathbf{x}})$, is given by $\operatorname{deg}(f(\underline{\mathbf{x}}))=\lceil n / 3\rceil+1$.

Proof. According to Fact 1 the degree of $f(\underline{\mathbf{x}})$ is equal to the algebraic degree of $f(x)$. From the definition, the algebraic degree of $f(x)$ is determined by the largest Hamming weight among the integers appeared in the set $I$ in Fact 4 . We can easily verify that $k+1$ for both $n=3 k-1$ and $n=3 k-2$ is such number. Thus the assertion is established. (See the full paper, for a detailed proof.)

Applying the Siegenthaler inequality [18] and Theorem [7, the following corollary is immediate.

Corollary 3. For a given $W G$ transformation regarded as a Boolean function in $n$ variables, $r$, the order of the resilient property of the function, is bounded by the following inequality $r \leq n-\lceil n / 3\rceil$. 


\section{Example}

In this section, we will give an example to illustrate the randomness properties of WG transformations regarded as both WG sequences and Boolean functions, which we obtained in the previous sections. Let $\mathbb{F}_{2^{7}}$ be defined by a primitive polynomial $h(x)=x^{7}+x+1$. Let $\alpha$ be a root of $h(x)$. Then $\alpha$ is a primitive element of $\mathbb{F}_{2^{7}}$. Since $n=7$, then $k=3$, and

$$
q_{1}=5, q_{2}=21, q_{3}=13, \text { and } q_{4}=29 \text { and } g(x)=x+x^{5}+x^{21}+x^{13}+x^{29}
$$

So, the WG transformation is

$$
f(x)=\operatorname{Tr}(g(x+1)+1)=\operatorname{Tr}\left(x+x^{3}+x^{7}+x^{19}+x^{29}\right) .
$$

A. Sequence Aspects of the WG transformation:

We obtain a WG sequence $\underline{\mathbf{b}}=\left\{b_{i}\right\}$ as follows:

$$
\begin{aligned}
\underline{\mathbf{b}}= & 1000000101000011011100010100101100101010000101100010010111011011 \\
& 000110011101100100000110001111010101110100100111111100111101111
\end{aligned}
$$

where $b_{i}=f\left(\alpha^{i}\right), i=0,1, \cdots$. The WG sequence $\underline{\mathbf{b}}$ has the Balance property, ideal 2-tuple distribution, 2-level auto correlation, 3-valued cross correlation with an m-sequence defined by $\left\{a_{i}\right\}$ where $a_{i}=\operatorname{Tr}\left(\alpha^{i}\right), i=0,1, \cdots$ which belongs to the set $\{-1,15,-17\}$, the Hadamard transform spectrum belonging to the set $\{0, \pm 16\}$ and linear span 35 .

B. Boolean Function Aspects of the WG transformation:

Using the polynomial basis $\left(1, \alpha, \alpha^{2}, \alpha^{3}, \alpha^{4}, \alpha^{5}, \alpha^{6}\right)$, where $\alpha^{7}+\alpha+1=0$, the algebraic normal form of the Boolean function that corresponds to $f(x)$ is given by

$x_{0} \oplus x_{1} x_{3} \oplus x_{0} x_{1} x_{3} \oplus x_{2} x_{3} \oplus x_{0} x_{2} x_{3} \oplus x_{1} x_{2} x_{3} \oplus x_{0} x_{3} x_{4} \oplus x_{2} x_{3} x_{4} \oplus x_{0} x_{5} \oplus x_{0} x_{1} x_{5} \oplus$ $x_{0} x_{2} x_{5} \oplus x_{1} x_{2} x_{5} \oplus x_{3} x_{5} \oplus x_{1} x_{3} x_{5} \oplus x_{1} x_{4} x_{5} \oplus x_{2} x_{4} x_{5} \oplus x_{2} x_{3} x_{4} x_{5} \oplus x_{1} x_{6} \oplus x_{2} x_{6} \oplus$ $x_{0} x_{2} x_{6} \oplus x_{0} x_{3} x_{6} \oplus x_{4} x_{6} \oplus x_{0} x_{4} x_{6} \oplus$

$x_{2} x_{4} x_{6} \oplus x_{1} x_{3} x_{4} x_{6} \oplus x_{0} x_{5} x_{6} \oplus x_{1} x_{5} x_{6} \oplus x_{1} x_{2} x_{5} x_{6} \oplus x_{0} x_{3} x_{5} x_{6}$.

Changing the basis using the following basis conversion matrix

$$
\left(\begin{array}{l}
\beta_{0} \\
\beta_{1} \\
\beta_{2} \\
\beta_{3} \\
\beta_{4} \\
\beta_{5} \\
\beta_{6}
\end{array}\right)=\left(\begin{array}{lllllll}
1 & 1 & 1 & 0 & 0 & 0 & 1 \\
0 & 1 & 0 & 0 & 0 & 0 & 0 \\
0 & 0 & 1 & 1 & 1 & 0 & 1 \\
0 & 0 & 0 & 1 & 0 & 1 & 0 \\
0 & 0 & 0 & 0 & 1 & 0 & 0 \\
0 & 0 & 0 & 0 & 0 & 1 & 0 \\
0 & 0 & 0 & 0 & 0 & 0 & 1
\end{array}\right)\left(\begin{array}{c}
1 \\
\alpha \\
\alpha^{2} \\
\alpha^{3} \\
\alpha^{4} \\
\alpha^{5} \\
\alpha^{6}
\end{array}\right)
$$

we obtain

$x_{0} \oplus x_{1} \oplus x_{2} \oplus x_{3} \oplus x_{0} x_{3} \oplus x_{0} x_{1} x_{3} \oplus x_{2} x_{3} \oplus x_{0} x_{2} x_{3} \oplus x_{1} x_{2} x_{3} \oplus x_{3} x_{4} \oplus x_{1} x_{3} x_{4} \oplus$ 
$x_{2} x_{3} x_{4} \oplus x_{5} \oplus x_{0} x_{5} \oplus x_{1} x_{5} \oplus x_{1} x_{3} x_{5} \oplus x_{0} x_{4} x_{5} \oplus x_{3} x_{4} x_{5} \oplus x_{2} x_{3} x_{4} x_{5} \oplus x_{6} \oplus x_{0} x_{6} \oplus$ $x_{0} x_{2} x_{6} \oplus x_{1} x_{2} x_{6} \oplus x_{0} x_{3} x_{6} \oplus$

$x_{1} x_{3} x_{6} \oplus x_{2} x_{4} x_{6} \oplus x_{1} x_{3} x_{4} x_{6} \oplus x_{5} x_{6} \oplus x_{0} x_{5} x_{6} \oplus x_{1} x_{5} x_{6} \oplus x_{2} x_{5} x_{6} \oplus x_{1} x_{2} x_{5} x_{6} \oplus$ $x_{3} x_{5} x_{6} \oplus x_{0} x_{3} x_{5} x_{6} \oplus x_{2} x_{3} x_{5} x_{6} \oplus x_{3} x_{4} x_{5} x_{6}$

which is 1-resilient function with nonlinearity 56 , algebraic degree 4 and linear span 35 .

\section{Conclusion}

We provide a table which contains the profiles that we obtained in previous sections as a conclusion of this paper.

Table 1. Profiles of WG transformations

\begin{tabular}{|c|c|c|c|c|}
\hline $\begin{array}{l}\text { WG Sequences } \\
\text { profile }\end{array}$ & WG Sequence & & $\begin{array}{c}\text { WG Trans. } \\
\text { as Boolean Func. }\end{array}$ & $\begin{array}{c}\text { WG Trans. Boolean } \\
\text { Profile }\end{array}$ \\
\hline $2^{n}-1$ & Period & $\leftrightarrow$ & Boolean & $n$ variables \\
\hline Yes & Balance & $\leftrightarrow$ & Balance & Yes \\
\hline Yes & 2-tuple distribution & & $\mathrm{NC}$ & \\
\hline 2-level & Auto correlation & & $\mathrm{NC}$ & \\
\hline $\begin{array}{c}\left\{-1,-1 \pm 2^{\frac{n+1}{2}}\right\}, n \text { odd } \\
\text { optimal w.r.t. } \\
\text { the Welch bound, }\end{array}$ & $\begin{array}{l}\text { cross correlation } \\
\text { with m-sequences }\end{array}$ & $\leftrightarrow$ & Non-linearity & $2^{n-1}-2^{\frac{n-1}{2}}, n$ odd \\
\hline $0, \pm 2^{(n+1) / 2}, n$ odd & Hadamard tran & isfo & m spectrum & $0, \pm 2^{(n+1) / 2}, n$ odd \\
\hline $\begin{array}{c}n\left(2^{|n / 3|}-3\right) \\
\text { increases } \\
\text { exponentially in } n\end{array}$ & Linear span & $\leftrightarrow$ & Linear span & $\begin{array}{c}n\left(2^{|n / 3|}-3\right) \\
\text { increases } \\
\text { exponentially in } n\end{array}$ \\
\hline & $\begin{array}{l}\mathrm{NC} \\
\mathrm{NC}\end{array}$ & & $\begin{array}{c}\text { Degree } \\
r \text {-resilient }\end{array}$ & $\begin{array}{c}\lceil n / 3\rceil+1 \\
r: 1 \leq r \leq n-\lceil n / 3\rceil\end{array}$ \\
\hline easy* & \multicolumn{3}{|c|}{ Implementation } & easy \\
\hline $\begin{array}{l}\text { Ideal candidates for } \\
\text { combining functions } \\
\text { Pseudo-random } \\
\text { sequence generators }\end{array}$ & \multicolumn{3}{|c|}{ Applications } & $\begin{array}{c}\text { Ideal candidates for } \\
\text { combining functions } \\
\text { or filtering functions } \\
\text { operating on } \\
\text { a set of LFSRs }\end{array}$ \\
\hline
\end{tabular}

Notations used in Table 1:

- NC means that there is no corresponding concept between them.

- *: There are two methods to implement WG sequences. One is to use 5 LSFRs together with a table look-up (Lemma 1 method). The other is to use a finite field configuration. The complexity of implementation of WG sequences by using the finite field configuration only depends on evaluation of four exponentiations listed in Section 2. Especially, it only depends on the evaluating the exponents $q_{3}$ and $q_{4}$ where each of them has $k-1$ consecutive 1 's. We will discuss how to efficiently compute these two exponentiations at a separate paper. (Note. Implementation of the trace function has no cost.)

\section{References}

1. Xingong Chang, Zongduo Dai and Guang Gong, Some cryptographic properties of exponential functions, Advances in Cryptology-AsiaCrypt'94, Lecture Notes in Computer Science, No. 917, Springer-Verlag, 1994, pp. 415-418. 
2. John Dillon, Multiplicative difference sets via additive characters, Designs, Codes and Cryptography. 17 (1999), no. 1-3, 225-235.

3. H. Dobbertin, Kasami power functions, permutation polynomials and cyclic difference sets, Proceedings of the NATO-A.S.I. Workshop "Difference sets, sequences and their correlation properties", Bad Windsheim, August 3-14, 1998, Kluwer, Dordrecht, pp. 133-158, 1999.

4. J. Dillon and H. Dobbertin, New cyclic difference sets with Springer parameters, preprint, August 1999.

5. S.W. Golomb, Shift Register Sequences, Revised Edition, Aegean Park Press, 1982, pp. 39.

6. G. Gong, P. Gaal and S.W. Golomb, A suspected infinity class of cyclic Hadamard difference sets, the Proceedings of 1997 IEEE Information Theory Workshop, July 6-12, 1997, Longyearbyen, Svalbard, Norway.

7. G. Gong and A. M. Youssef, On Welch-Gong Transformation Sequence Generators, Technical report, University of Waterloo, CORR 2000-30, May 2000, http://www.cacr.math.uwaterloo.ca/techreports/2000.

8. R. Gold, Maximal recursive sequences with 3-valued recursive cross-correlation functions, IEEE Trans. on Inform. Theory, January 1968, pp. 154-156.

9. G. Gong, Lecture notes, http://cacr.math.uwaterloo.ca/ ggong/CO739x/739xcover.html

10. G. Gong and S.W. Golomb, Transform domain analysis of DES, IEEE Trans. on Inform. Theory, vol. 45, No.6, September 1999, pp. 2065-2073.

11. R. Lidl and H. Niederreiter, Finite Fields, Encyclopedia of Mathematics and its Applications, Volume 20, Addison-Wesley, 1983.

12. J.S. No, S.W. Golomb, G. Gong, H.K. Lee, and P. Gaal, New binary pseudorandom sequences of period $2^{n}-1$ with ideal autocorrelation, IEEE Trans. on Inform. Theory, vol. 44, No. 2, March 1998, pp.814-817.

13. F.J. MacWilliams and N.J.A. Sloane, The Theory of Error-Correcting Codes, North-Holland Publishing Company, 1977.

14. A. Maschietti, Difference sets and hyperovals, Designs, Codes and Cryptography, 14, pp. 89-98, 1998.

15. J. M. Massey, Shift register synthesis and $B C H$ decoding, IEEE transactions on Information Theory. Vol. 15, no. 1, pp. 122-127, January, 1969.

16. Jong-Seon No, Hagong Chung and Min-Seon Yin, Binary pseudo-random sequences of period $2^{m}-1$ with ideal autocorrelation generated by the polynomial $z^{d}+(z+1)^{d}$, IEEE Trans. Inform. Theory, vol. 44, no. 3, 1998, pp.1278-1282.

17. R.A. Rueppel, Analysis and Design of Stream Ciphers, Springe-Verlag, 1986, pp.53.

18. T. Siegenthaler, Correlation-immunity of nonlinear combining functions for cryptographic applications, IEEE Trans. on Inform. Theory, vol. IT-30, No. 5, September 1984, pp.776-780.

19. A.F. Webster and S.E. Tavares, On the design of S-boxes, Advances in CryptologyCrypto'85, Lecture Notes in Computer Science, No. 218, Springer-Verlag, 1985, pp. 523-534.

20. Guozheng Xiao and James Massey, A spectral characterization of correlation immune combining functions, IEEE Trans. on Inform. Theory, vol. 34, No. 3, May 1988, pp.569-571.

21. A. M. Youssef and G. Gong, On the interpolation attacks on block ciphers, Proceedings of Fast Software Encryption 2000 , April 13-14, 2000, New York, Lecture Notes in computer science, to appear. 